\title{
Erratum to: New high-strength Ti-Al-V-Mo alloy: from high-throughput composition design to mechanical properties
}

\author{
Di W $u^{1,2)}$,Wan-lin Wang ${ }^{1)}$, Li-gang Zhang ${ }^{2,3)}$,Zhen-yu Wang ${ }^{2)}$, Ke-chao Zhou ${ }^{4)}$, and Li-bin Liiu ${ }^{2,3)}$ \\ 1) School of Metallurgy and Environment, Central South University, Changsha 410083, China \\ 2) School of Material Science and Engineering, Central South University, Changsha 410083, China \\ 3) Key Laboratory of Non-Ferrous Metallic Materials Science and Engineering, Ministry of Education, Changsha 410083, China \\ 4) State Key Laboratory of Powder Metallurgy, Changsha 410083, China
}

Erratum to: International Journal of Minerals, Metallurgy and Materials

Volume 26, Number 9, September 2019, Page 1151

https://doi.org/10.1007/s12613-019-1854-1

The original version of this article unfortunately contained a mistake. The presentation of Fig. 11 was incorrect. The correct version is given below:
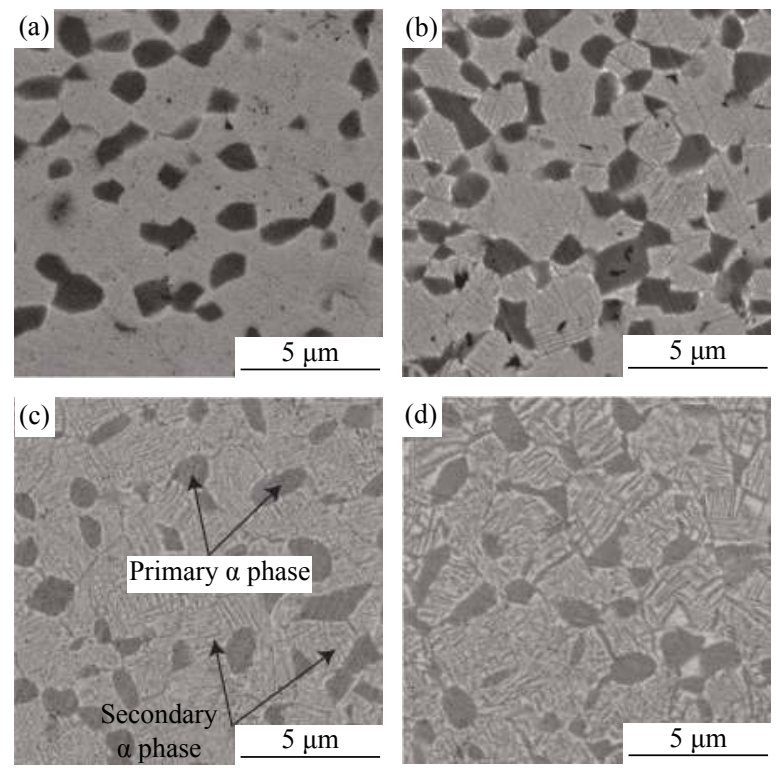

The online version of the original article can be found at https://doi.org/10.1007/s12613-019-1854-1

Corresponding authors: Ke-chao Zhou E-mail: zhoukechao@csu.edu.cn; Li-bin LiuＥ-mail: lbliu@csu.edu.cn (C) University of Science and Technology Beijing and Springer-Verlag GmbH Germany, part of Springer Nature 2020 